\title{
Prevalencia de síndrome de Down en CEHANI-ESE, San Juan de Pasto Colombia. 1998-2003
}

\author{
Iván Hernández Ramírez ${ }^{1,2,3}$, Ruben Dario Manrique Hernández ${ }^{1}$ \\ 1CESUN: Instituto de Ciencias de la Salud-Medellín y Universidad de Nariño, Especialización en Epidemiología; \\ ${ }^{2}$ Fundación Universitaria San Martín sede San Juan de Pasto. \\ ${ }^{3}$ Convenio Interinstitucional FUSM-CEHANI, (Centro de Habilitación del Niño) San Juan de Pasto. \\ Correspondencia: ivanher6@hotmail.com \\ Recibido: 12-04-2006 Aceptado:08-06-2006.
}

\begin{abstract}
Resumen
Un estudio colaborativo para América Latina sobre malformaciones congénitas (ECLAM) detectó una alta incidencia de síndrome de Down en Sur América, en Colombia no existen reportes. Con el objetivo de analizar la prevalencia de síndrome de Down entre 1998 y 2003 en el Centro Regional de Referencia (CEHANIESE) de San Juan de Pasto - Colombia, se llevó a cabo el presente estudio, utilizando la información recolectada mediante la base de datos de almacenamiento de historias clínicas ECLAM, que permite el registro de nacidos vivos con malformaciones atendidos en CEHANI, dichos datos fueron comparados contra el total de nacimientos del mismo sexho en la población. Como resultado se encontró que durante el tiempo de estudio, se registraron 49 casos de síndrome de Down, 5 casos por cada 10.000 nacidos vivos, una cifra mas baja de la esperada. Con respecto a la edad, el rango entre 30 y 34 años presentó la misma frecuencia de ocurrencia del SD que en mujeres mayores de 35 años. En relación con el sexo, se identificó un mayor número de niñas afectadas en comparación con el número de niños y la tasa de nacimiento por sexo.
\end{abstract}

Palabras claves: síndrome de Down, prevalencia, genética, base de datos.

\begin{abstract}
Prevalence of Down syndrome in the CEHANI-ESE in San Juan de Pasto Colombia. 1998-2003

Background: The Latin American Collaborative Study for Congenital Malformations (ECLAMC) has detected a higher incidence of Down syndrome in South America. In Colombia there are not reports about it. Aim: To analyze the prevalence rates of Down syndrome between 1998 and 2003 at the CEHANI-ESE Regional Center of reference in San Juan de Pasto located on the south of the Country. Material and methods: The information obtained by ECLAMC was used. This program, using a secondary data base (clinic history) as a method, registers all the patients who were born with congenital malformations attended in CEHANI, to be compared with the total of births of the same sex, in the population. Results: During the study period, 49 cases of Down syndrome were registered, with a mean rate of 5 per 10,000 live births, lower than expected. The rate variations along the years suggest a cyclic change, with a hemicycle of five years. Conclusions: In this Reference Center, mothers between 30 and 35 years of age, have the same risk of having a child with
\end{abstract}


Down syndrome, most of the births with Down syndrome belong to mothers under 35 years old. At the moment of the birth, there were more girls than boys with Down syndrome, compared with a higher birth of boys than girls.

Key Words: Down syndrome, prevalence, genetics, secondary data base

\section{Introducción}

El síndrome de Down (SD) dentro de las malformaciones congénitas externas, es el trastorno cromosómico más frecuente entre los países que reportan al Directorio Internacional de Defectos Congénitos: International Clearing House for Birth Defects Monitoring "ICDBMS” (Clearinghouse) (1$5)$, en donde es posible observar cifras tan elevadas en la prevalencia al nacimiento $(\mathrm{PN})$ como las de la región Suraméricana, con 15,7 Síndrome de Down (SD) por 10.000 nacidos vivos (NV) o cifras tan bajas como 4,2 SD por 10.000 NV en Japón $(1,4-5)$.

Un estudio desarrollado en Cali-Colombia y publicado en la Revista Colombia Médica en 1996 reporta para el quinquenio 1991 a 1995 una $\mathrm{PN}$ de $15 \mathrm{SD}$ en $10.000 \mathrm{NV}$, después de analizar la información proveniente de cinco laboratorios de citogenética en la ciudad (4).

El Municipio de San Juan de Pasto, Ciudad Capital del Departamento de Nariño se encuentra situado en el Valle de Atriz a 795 kilómetros al suroccidente de la Capital de la República, cuenta con aproximadamente 400.000 habitantes (año 2003) y un promedio de nacimientos por año de 19.888 (51\% de hombres y $49 \%$ de mujeres), tendencia observada en el quinquenio comprendido entre 1999 y 2003.

El Centro de Habilitación del Niño "CEHANI", Institución de referencia en la región, cumple con una importante función en la sociedad Nariñense, orientando el manejo del "niño especial" (todos aquellos niños con discapacidad cognoscitiva), pero desafortunadamente, no llegan allí todos los pacientes con SD, debido a múltiples factores que influyen sobre los familiares de los pacientes como la sobreprotección a estos niños y/o el desconocimiento de la ayuda prestada por el centro, entre otros. La base de datos secundarios presente en la Institución, aunque tiene información debidamente archivada por más de una década, no registra a todos los SD presentes en la Ciudad, situación que se convierte en una limitación del presente estudio.

En el primer semestre del 2003, se realizó un estudio exploratorio sobre la base de datos secundaria del CEHANI-ESE, para establecer la calidad de la información de las historias clínicas, para ello se tomaron 22 historias clínicas correspondientes a pacientes con SD cuyas registros correspondían a los últimos dos años (2001 y 2002), dicha observación conllevó a la construcción de un instrumento para obtener datos necesarios en la alimentación de la base de datos del Programa de Registro del CEHANI-ESE.

La observación permitió al cruzar la información obtenida hasta el momento: nacimientos entre el 2001 y el 2002 (38.256 según cifras del DANE/2003 para Pasto), frente a los registros obtenidos en el CEHANIESE, en el mismo periodo: 22 pacientes con SD, así como conocer la incidencia del SD: 1/1000 nacimientos durante el periodo referente. Sin embargo, al ampliar el periodo de tiempo estudiado los valores cambiaron notablemente.

La Fundación Universitaria San Martin F.U.S.M., dentro de la función que cumple frente a la comunidad y en especial por su misión dirigida a la formación de médicos generales con tendencia social, comunitaria y familiar, decide proyectarse con el área de formación en ciencias básicas, mediante un proyecto docente, que implica convenio interinstitucionales, con el ánimo de ofrecer elementos útiles en el diagnóstico inmediato del SD, su registro y control epidemiológico, al igual que un Programa de información accesible para quienes requieran conocer más sobre el $\mathrm{SD}$, en la región. 
NOVA - PUBLICACIÓN CIENTÍFICA ISSN:1794-2470 VOL.4 No. 5 ENERO - JUNIO DE 2006:1-116

En este estudio se muestra la prevalencia del Síndrome de Down en el CEHANI-ESE durante el quinquenio comprendido entre 1998 y 2003 con la metodología propuesta en la estadística descriptiva, considerando el tamaño reducido de casos observados, describiendo además, algunas variables relacionadas con la ocurrencia del Síndrome de Down como el bajo estrato social, el patrón reproductivo alterado y la edad materna.

\section{Materiales y métodos}

La información obtenida de los registros de CEHANI-ESE y el DANE para el Municipio de San Juan de Pasto en el quinquenio comprendido entre 1998 y 2003, fueron recolectados mediante un instrumento en forma de tabla en donde se registra la información proveniente de las historias clínicas archivadas en la institución, la metodología consiste entonces, en la recolección de datos obtenidos de las fuentes de datos secundarias como son las historias clínicas obtenidas de los archivos del CEHANI-ESE, el mecanismo establece la condición retrospectiva y el estudio de cohortes sucesivas durante el periodo establecido de cinco años, que permite el análisis de la frecuencia con que se presentaron los casos de Síndrome de Down en dicha entidad de carácter referencial para el municipio, la evaluación se enmarco dentro de la estadísticas descriptiva simple.

Se obtuvieron incidencias año tras año, durante el quinquenio 1998-2003 y al final del periodo se calculó la prevalencia total encontrada correspondiente a 5 SD por $10.000 \mathrm{NV}$ en el periodo, y por sexo: $5.5 \mathrm{SD}$ por $10000 \mathrm{NV}$ en niñas y $4 \mathrm{SD}$ por $10000 \mathrm{NV}$ en niños. La prevalencia observada para Sur América era de 15 SD por $10000 \mathrm{NV}$.

El análisis univariado de la edad materna y la edad paterna se puede observar en la Tabla 1.

\section{Resultados}

La Tabla 2 muestra la distribución anual de nacidos vivos (NV) y los síndrome de Down registrados en el CEHANI-ESE durante el periodo 1998-2003. La Tabla 2, resume la evidencia que sustenta el bajo número de registros con Síndrome de Down en el CEHANI-ESE durante el quinquenio estudiado, observando en un total de nacimientos entre hombres y mujeres de 99423 que tan solo se registraron en la Institución 49 SD. De igual manera, analizando por sexo el $51 \%$ de los NV eran hombres y el $49 \%$ eran mujeres, mientras que en comparación con $\mathrm{SD}$, el porcentaje de hombres con SD fue de $41 \%$ y el de mujeres con SD fue de $59 \%$.

El cálculo de la incidencia año tras año durante el quinquenio 1998-2003 se muestra en la Tabla 3. en ella, se calcula además la prevalencia total y la prevalencia por género, observando fluctuaciones notables entre los valores obtenidos, así pues, la incidencia más alta se observó en el primer año (1998) 21 casos de SD por $10000 \mathrm{NV}$. Al final de los 5 años (al final del periodo) la prevalencia total fue de 5 casos de SD por $10000 \mathrm{NV}$.

La Tabla 4 muestra la evidencia sobre el riesgo observado en el rango de edad materna 30-34 años cuyo comportamiento fue igual con respecto al rango de 35-39 años de edad materna, se pudo ver una tendencia bimodal cuya frecuencia en ambos casos fue de 13 casos de SD, lo cual sugiere igual riesgo para ambos rangos, es decir un mayor cuidado para las mujeres embarazadas desde los 30 años $(6,7,8,9)$.

La Tabla 5. muestra el comportamiento del patrón reproductivo (edad de la pareja) $(6,10,11,13)$ de los 49 casos registrados analizados en el estudio, aunque los casos extremos son pocos 6 de 49, las parejas adultas con riesgo que se atreven a tener hijos son muchos más, alrededor de 15 de 49.

La tabla 6. muestra la distribución de los casos según el estrato social dependiendo de la afiliación al SISBEN, el 88\% de los casos distribuidos en los estratos 1, 2 y 3, lo cual indica una baja estratificación (12).

www.unicolmayor.edu.co 
Tabla 1. Análisis Univariado de la edad materna y paterna

EDAD MATERNA

$X=30,63$ años $(30-34)$

$\mathrm{Me}=32(30-34)$

Mo $=32(30-34) 37(35-39)$

$\mathrm{S}^{2}=70,3548$

$S=8,3877$

\section{EDAD PATERNA}

$X=32,65$ años (30-34)

$\mathrm{Me}=34(30-34)$

Mo $=35(35-39)$

$\mathrm{S}^{2}=86.1449$

$S=9,2814$

\section{EDAD MATERNA}

$\mathrm{CV}=27,62$

$X_{\max }=45(45-49)$

$X_{\min }=12(10-14)$

I Cuartil 12.5ésima observación ordenada

II Cuartil 25ésima observación ordenada

III Cuartil 37.5ésima observación ordenada

IQR 25 pequeña variabilidad

$\mathrm{R} \quad 33$ (dato mayor - d. menor)

100(IQR/R) $\quad 75.75 \%$ toda la amplitud
EDAD PATERNA

$\begin{array}{ll}\mathrm{CV}= & 28,42 \\ \mathrm{X}_{\max }= & 56(50>) \\ \mathrm{X}_{\min }= & 15(15-19) \\ \text { I Cuartil } & 12.5 \text { ésima } 0.0 . \\ \text { II Cuartil } & 25 \text { ésima } 0.0 . \\ \text { III Cuartil } & 37.5 \text { ésima } 0.0 . \\ \text { IQR } & 25 \mathrm{pv} \\ \mathrm{R} & 41 \\ 100(\mathrm{IQR} / \mathrm{R}) & 60.97 \% \text { amplitud }\end{array}$

Tabla 2. Casos de SD según historias clínicas CEHANI por género y fecha de atención quinquenio 1999-2003, frente a los nacimientos por área DANE.

\begin{tabular}{|c|c|c|c|c|c|c|}
\hline \multirow[t]{2}{*}{ Año } & \multicolumn{2}{|c|}{ No de hombres } & \multicolumn{2}{|c|}{ No de mujeres } & \multicolumn{2}{|c|}{ Total $\mathrm{h} / \mathrm{m}$} \\
\hline & SD & NV & SD & NV & SD & NV \\
\hline 1999 & 9 & 11786 & 12 & 11212 & 21 & 22998 \\
\hline 2000 & 3 & 9006 & 1 & 8723 & 4 & 17729 \\
\hline 2001 & 5 & 8987 & 5 & 8707 & 10 & 17694 \\
\hline 2002 & 2 & 10462 & 10 & 10100 & 12 & 20562 \\
\hline 2003 & 1 & 10412 & 1 & 10028 & 2 & 20440 \\
\hline Quinquenio & 20 & 50653 & 29 & 48770 & 49 & 99423 \\
\hline
\end{tabular}


Tabla 3. Incidencia del SD por género durante el quinquenio 1999-2003

\begin{tabular}{|c|c|c|c|c|c|c|c|c|c|}
\hline \multirow[t]{2}{*}{ Año } & \multicolumn{3}{|c|}{$\underline{\text { Hombres }}$} & \multicolumn{3}{|c|}{$\underline{\text { Mujeres }}$} & \multirow[b]{2}{*}{ NV } & \multicolumn{2}{|c|}{ Total } \\
\hline & NV $\leqq$ & SD & I x 10000 & NV & $S D$ & Ix10000 & & SD & $1 \times 10000$ \\
\hline 1999 & 11786 & 9 & 8 & 11212 & 12 & 10 & 22998 & 21 & 10 \\
\hline 2000 & 9006 & 3 & 3 & 8723 & 1 & 2 & 17729 & 4 & 2 \\
\hline 2001 & 8987 & 5 & 6 & 8707 & 5 & 6 & 17694 & 10 & 4 \\
\hline 2002 & 10462 & 2 & 2 & 10100 & 10 & 10 & 20562 & 12 & 6 \\
\hline 2003 & 10412 & 1 & 1 & 10028 & 1 & 1 & 20440 & 2 & 2 \\
\hline Total & 50653 & 20 & 4 & 48770 & 29 & 5.5 & 99423 & 49 & 5 \\
\hline
\end{tabular}

Tabla 4. Distribución porcentual de la edad materna con hijos SD Observada. CEHANI - Pasto.

Edad de la Madre

(años)
No. Maternas
Porcentaje

$(\%)$

$\begin{array}{ccc}10-14 & 1 & 2 \\ 15-19 & 8 & 16.3 \\ 20-24 & 4 & 8.2 \\ 25-29 & 3 & 6.1 \\ 30-34 & 13 & 26.5 \\ 35-39 & 13 & 26.5 \\ 40-44 & 6 & 12.2 \\ 45-49 & 1 & 2 \\ \text { Total } & 49 & 100\end{array}$

\section{Discusión}

Se observó con las evidencias epidemiológicas presentadas en este estudio en primer lugar, que la prevalencia del Síndrome de Down en CEHANI-ESE, es mucho más baja que la observada en los datos para Sur América de CLEARINGHOUSE $(4,5,13,16)$, lo cual pudo deberse, a que esta institución a pesar de ser referencial para el Municipio, no atiende todos los casos de SD en la población.
Con respecto a la edad, el rango entre 30 y 34 años presentó la misma frecuencia de ocurrencia del $\mathrm{SD}$ que la de 35 a 39 años lo cual sugiere un riesgo potencial a partir de los 30 años en mujeres gestantes. Se evidencia así que la distribución de la edad materna, respecto a un mayor riesgo, es mucho menor que la esperada $(5,15,16)$. Debido a que en ninguno de los casos se hizo un diagnóstico prenatal, no se pudieron analizar otros datos. Esta situación esta relacionada 
Tabla 5. Edades en años cumplidos de madres y padres por pareja en el momento de nacer el SD, elementos muestrales-CEHANI. quinquenio 1999-2003

\begin{tabular}{|llclclcl|}
\hline Mamá & Papá & Mamá & Papá & Mamá & Papá & Mamá & Papá \\
\hline 32 & 28 & 27 & 27 & 40 & 56 & 32 & 30 \\
33 & 38 & 45 & 43 & 18 & 21 & 32 & 31 \\
17 & 29 & 30 & 32 & 37 & 35 & 12 & 15 \\
27 & 17 & 24 & 31 & 32 & 34 & 37 & 35 \\
16 & 18 & 37 & 40 & 39 & 39 & 32 & 34 \\
37 & 36 & 39 & 39 & 31 & 36 & 37 & 35 \\
32 & 32 & 42 & 47 & 43 & 47 & 19 & 21 \\
31 & 36 & 25 & 25 & 21 & 28 & 18 & 21 \\
37 & 35 & 34 & 52 & 34 & 30 & 33 & 20 \\
39 & 43 & 41 & 46 & 16 & 19 & 39 & 36 \\
28 & 35 & 18 & 18 & 23 & 23 & 35 & 35 \\
32 & 38 & 40 & 41 & 22 & 32 & 37 & 39 \\
19 & 22 & & & & & & \\
\hline
\end{tabular}

Tabla 6. Distribución porcentual del grupo con SD por afiliación al SGSSS (SISBEN). CEHANI - Pasto

\begin{tabular}{|cccccc|}
\hline Afiliación SGSSS & Hombres & $\%$ & Mujeres & $\%$ \\
SI & 17 & 85 & 26 & 90 \\
NO & 3 & 15 & 3 & 10 \\
\hline
\end{tabular}

con el bajo estrato social que presentan los beneficiarios del Régimen o Sistema General de Seguridad Social en Salud (SISBEN) y la falta de cubrimiento por el sistema para estos exámenes.

Las diferencias en edades y la decisión de tener hijos sobrepasando la edad de riesgo obedecen en general, a falta de educación sobre el particular (5-7). Se plantea la necesidad de identificar las posibles causas genéticas del origen del SD en las parejas jóvenes, la cual puede ser de tipo familiar (12-16), pero se requiere de la intervención del Estado mediante los organismos regionales de salud, que permitan llevar a cabo estudios citogenéticos para el diagnóstico efectivo del síndrome, así como el análisis dermatoglífico $(3,7,12,14)$ elemento indispensable para caracterización y junto con el cariotipo, lograr un diagnóstico preciso del SD $(3,7)$.

\section{Agradecimientos}

Agradezco el apoyo ofrecido por la Gerente del Centro de Habilitación del Niño CEHANI-ESE San Juan de Pasto-Nariño, Dra Maria Isabel Arroyo de Fijten, al igual que la ayuda prestada por los estudiantes que hasta la fecha han cursado la Asignatura Genética del Programa de Medicina de la Fundación Universitaria San Martín, al personal Docente y Administrativo en cabeza del Dr Juan Carlos Montenegro Cardona, que facilitó el desarrollo del convenio Interinstitucional CEHANI-FUSM Sede Pasto, así 
como al Dr Ruben Dario Manrique H. Docente del CES Instituto de Ciencias de la Salud-Medellín por sus asesoría y conocimiento y a los Docentes y Directivos del convenio CESUN: CES y Universidad de Nariño bajo la Dirección de la Dra. Cristina Cerón Sousa, por la orientación y colaboración prestada.

\section{Referencias}

1. Ferrero M, Alonso F, Cedán I, Roca J, Perez A, Estévez M. Tendencias del Síndrome de Down en Cuba, su relación con la edad materna y tasa de fecundidad. Revista Cubana de Pediatría, 1998; 70(3): 141-147

2. García H, Salguero G, Moreno J, Arteaga C, Giraldo A. Frecuencia de anomalías congénitas en el Instituto Materno Infantil de Bogotá. Biomédica articulo original 2003; 23: 161-172

3. Thompsom J.S, Thompsom M. W. Genetics in Medicine, $4^{\text {th }}$ edition. Philadelphia: WB Saunders Co; 1991

4. Ramírez E, Isaza C, Gutiérrez M. La incidencia del Síndrome de Down en Cali. Colombia Médica 1996; 27: 138-142

5. Luna L, Babinsky B, Mutchinick OM. Síndrome de Down: tendencia en el tiempo (1978-1999) y riesgo de ocurrencia por quinquenio, bienio y año de edad materna. InstitutoNacional de Ciencias Médicas y Nutrición Salvador Zubirán, México D.F.
6. O.P.S. Maternidad saludable. Diagnóstico Nacional de Salud Sexual y Reproductiva, OPS- Colombia, 1998

7. Thompsom J.S, Thompsom M. W. Genética Médica. Barcelona: Salvat Editores; 1975. Pgs. 138-162

8. Slater E, Cowie V. Genética de los Trastornos Mentales: Anomalías de los autosomas. Barcelona: Salvat Editores; 1974. Pgs. 325-345

9. Falconer D. Introducción a la Genética Cuantitativa: Endogamia. México D. F: CECSA; 1980. Pgs 295-389

10. Garber E D. Introducción a la Citogenética: Citogenética de Mamíferos. México D. F: CECSA; 1975. Pgs. 203-219

11. Strickberger M W. Genética: Cambios en la estructura de los cromosomas. $3^{\mathrm{a}}$ edición. Colombia: Omega; 1988. Pgs. 457-482

12. Niubó I, Cruz A, Ayuso G. Sistema computarizado de análisis y cálculo con dermatoglifos. Revista Cubana de Investigación en Biomedicina 1997; 16(1): 34-39

13. Ramírez E, Isaza C, Gutiérrez M. La incidencia del Síndrome de Down en Cali. Colombia Médica 1996; 27: 138-142

14. Penrose I.S. Memorandum on dermatoglyphic nomenclature. Birth Defects Original Article Series 1968; 4(3).

15. Wright S W, Day R W, Muller H, Weinhouse R. The frequency of trisomy and translocations in Down's syndrome. Jou Pediatric 1967; 70: 420-424

16. Hook E B. Estimates of maternal age-especific risk of a Down-syndrome birth in women aged 34-41. Lancet, 1976; 2: 334 